AN. MED INTERNA (Madrid) AN. MED. INTERNA (Madrid)
Vol. 17, N. ${ }^{\circ}$ 12, pp. 649-651, 2000

\section{Listeriosis: una infección poco frecuente en pacientes con VIH}

\author{
M. E. VALENCIA ORTEGA, A. ENRÍQUEZ CREGO*, F. LAGUNA CUESTA, \\ G. ORTEGA MILLÁN, J. M. GONZÁLEZ LAHOZ
}

Servicios de Enfermedades Infecciosas y *Microbiología. Hospital Carlos III. Madrid

LISTERIOSIS: AN INFREQUENT INFECTION IN PATIENTS WITH HIV

\section{RESUMEN}

Aunque la resistencia a la infección por Listeria monocytogenes requiere que la inmunidad celular este indemne, la listeriosis es una enfermedad poco frecuente en los pacientes VIH+ y existen únicamente alrededor de unos 50 casos publicados.

Se presentan los dos únicos enfermos $\mathrm{VIH}+$ con infección por $L$. monocytogenes atendidos en nuestro hospital desde el inicio de la epidemia del sida en 1981.

Caso 1: Varón VIH+ con 364 linfocitos CD4+ por $\mathrm{mm}^{3}$ que ingresó por cefalea occipital y fiebre. La tomografía axial computarizada cerebral fue normal y en el cultivo del líquido cefalorraquídeo creció $L$. monocytogenes. Se trató favorablemente con ampicilina y tobramicina.

Caso 2: Varón de 47 años VIH+ con 44 linfocitos CD4+/ $\mathrm{mm}^{3}$ y cirrosis hepática por VHC que ingresó por fiebre elevada y distensión abdominal. Realizaba profilaxis para PCP con pentamidina IV. Con el diagnóstico de peritonitis bacteriana espontánea se extrajeron 3 hemocultivos y se inició tratamiento con ceftriaxona. Desarrolló encefalopatía hepática y falleció a las $72 \mathrm{~h}$ del ingreso. Postmortem se recibió crecimiento de L. monocytogenes .

La listeriosis es una enfermedad poco frecuente en los enfermos VIH+, que esporádicamente produce cuadros de difícil diagnóstico, sobre todo en los enfermos que no realizan profilaxis para PCP con cotrimoxazol.

PALABRAS CLAVE: L. monocytogenes y VIH. Listeriosis y VIH. Bacteriemia por L. monocytogenes y VIH. Meningitis por L. monocytogenes y VIH.

\section{ABSTRACT}

Although resistance to Listeria monocytogenes infection requires intact T-cell mediated immunity, listeriosis is an infrequent problem in patients with HIV infection and only about 50 patients have been repor ted to date.

Only two patients with HIV and L. monocytogenes have been aten ded in our hospital since the beginning of aids epidemic in 1981.

Case 1: a man with HIV and $364 \mathrm{CD} 4+$ cells $/ \mathrm{mm}^{3}$ presented fever and occipital headache. The cerebral scan was normal and L. monocyto genes grew in licuor culture. He was outcome after tratment with ampici llin and tobramycin.

Case 2: a 47 years old man with HIV, $44 \mathrm{CD} 4+$ cells $/ \mathrm{mm}^{3}$ and hepa tic virus $C$ cirrhosis was admitted to the hospital because fever and abdominal distension. He was on mensual pentamidine prophylaxis for Pneumocystis carinii pneumonia (PCP). Bacterial peritonitis was diag nosed and the patient begun treatment with ceftriaxone. The patient dead 72 hours later with hepatic encepholopaty. Postmortem L. monocytoge nes grew.

Listeriosis is an infrequent disease in patients with HIV that causes difficult diagnostic problems, pricipally in patients without prophylaxis with cotrimoxazole for $P C P$.

KEY WORDS: L. monocytogenes and HIV. Listeriosis and HIV. L. monocytogenes bacteriemia and HIV. L. monocytogenes meningitis and $H I V$.

Valencia Ortega ME, Enríquez Crego A, Laguna Cuesta F, Ortega Millán G, González Lahoz J.M. Listeriosis: una infección poco frecuente en pacientes con VIH. An Med Interna (Madrid) 2000; 17: 649-651.

\section{INTRODUCCIÓN}

La Listeria monocytogenes es un germen ubicuo con puerta de entrada poco clara, aunque la vía gastrointestinal parece la más probable. Origina, fundamentalmente en pacientes con alguna causa de inmunodepresión, cuadros de bacteriemia y meningitis. En más del $50 \%$ de los casos se recoge el antecedente de un episodio de diarrea los días previos al inicio de la sintomatología neurológica (1).

Aunque la resistencia a la infección por L. monocytoge- nes requiere que la inmunidad celular este indemne (1,2), la listeriosis no es demasiado frecuente en los pacientes con infección por el VIH y existen poco más de 50 casos publicados (3). Otros factores que predisponen a esta infección son las edades extremas de la vida (recién nacido, anciano), el embarazo y la inmunodepresión de cualquier otro origen (4-6).

Se presentan a continuación los 2 únicos casos de listeriosis en pacientes con infección por VIH atendidos en un hospital de Madrid desde el inicio de la epidemia del sida en 1981.

Trabajo aceptado: 30 de Marzo de 1999

Correspondencia: $\mathrm{M}^{\mathrm{a}}$. Eulalia Valencia Ortega. Sector Músicos 14, 4 A. 28760 Tres Cantos (Madrid) 


\section{CASOS APORTADOS}

Caso 1: Varón de 32 años, ex-adicto a drogas por vía parenteral (ADVP), VIH negativo 3 años antes, que consultó por cefalea occipital, fiebre y dolor de espalda de 24 h de evolución. Refería, además, haber tenido diarrea y dolor abdominal que desaparecieron de forma espontánea una semana antes del ingreso. No tenía otros antecedentes de interés y no realizaba ningún tratamiento. En la exploración física se objetivó muguet, una temperatura axilar de $39^{\circ}$ y rigidez de nuca sin focalidad neurológica. El hemograma, la bioquímica sanguínea, el sistemático de orina, la radiografía de tórax y la tomografía axial computarizada cerebral fueron normales o presentaron alteraciones poco valorables. Se realizó una punción lumbar que mostró 2000 células por $\mathrm{mm}^{3}$ ( $80 \%$ polimorfonucleares), una glucosa de $38 \mathrm{mg} \%$ (plasmática $99 \mathrm{mg} \%$ ) y unas proteínas de 1,40 g/dL. Aunque inicialmente no estaba diagnosticado de infección por VIH, se sospechó por la existencia de muguet. Se confirmó por ELISA y Western-blot y el recuento de linfocitos $\mathrm{CD}^{+}$fue de 364 por $\mathrm{mm}^{3}$ $(14 \%)$. El cuadro fue interpretado como una meningitis, probablemente bacteriana, y se inició tratamiento con ampicilina y cefotaxima ambas administradas de forma intravenosa. En el cultivo del líquido cefalorraquídeo creció Listeria monocytogenes. La cefotaxima se sustituyó por tobramicina después de identificar el agente responsable. Tras 7 días de terapia apareció toxicodermia severa que condicionó retirada de la medicación. Ambos antibióticos fueron suspendidos iniciándose tratamiento con cotrimoxazol hasta comple$\operatorname{tar} 4$ semanas. La evolución fue favorable.

En la tabla I se observa la evolución de los parámetros del líquido cefalorraquídeo durante el tratamiento.

Caso 2: Varón de 47 años VIH (+) con 44 linfocitos CD4+ por $\mathrm{mm}^{3}$, trombopenia grave y cirrosis hepática con serología positiva para el virus de la hepatitis C (VHC) que ingresó por fiebre elevada, rectorragias, distensión abdominal y deterioro del estado general. Entre sus antecedentes destacaba alergia a las sulfamidas y haber padecido un episodio de candidiasis oral y neumonía por Pneumocystis carinii. Realizaba profilaxis con pentamidina intravenosa. Con el diagnóstico de probable peritonitis bacteriana espontánea se extrajeron 3 hemocultivos y se inició tratamiento con ceftriaxona. La situación clínica se deterioró progresivamente. Desarrolló encefalopatía hepática y hemorragia digestiva alta falleciendo a las $72 \mathrm{~h}$ del ingreso. Postmortem se recibió crecimiento de L. monocytogenes en los hemocultivos.

\section{DISCUSIÓN}

La L. monocytogenes es un germen gram positivo aerobio que origina un amplio espectro de enfermedades (4-11). En el adulto se detecta fundamentalmente en aquellos sujetos con alguna causa conocida de inmunosupresión como neoplasias, trasplantados, cirróticos o pacientes con infección por VIH (46). En estos últimos, suele aparecer cuando ya existe un diagnóstico de sida y/o los linfocitos CD4+ son inferiores a 200 por $\mathrm{mm}^{3}(2,3,6,7-12)$. Esto sucedió en el segundo caso en el que la evolución fue fatal en un corto periodo de tiempo. Sin embargo el paciente número 1 presentó un cuadro meníngeo cuando su inmunidad celular estaba todavía relativamente conservada (CD4+ >300 por $\mathrm{mm}^{3}$ ). La toma de cotrimoxazol de forma profiláctica en los enfermos con VIH y linfocitos CD4+ inferiores a 200 por $\mathrm{mm}^{3}$, probablemente influya en que la frecuencia de la listeriosis sea menor de la esperada. Así por ejemplo, ninguno de los 2 enfermos presentados realizaba profilaxis con cotrimoxazol.

En el caso del paciente $\mathrm{n}^{\circ} 1$, que cursó con un cuadro clínico compatible con meningitis, la rapidez de presentación y los hallazgos del líquido cefalorraquídeo (LCR) hacen pensar en una meningitis probablemente bacteriana. Dentro de éstas, las mas frecuentes en un varón joven, independientemente del VIH, son las producidas por Streptococcus pneumoniae, Neis seria meningitidis y Listeria monocytogenes (7). Realizando una tinción de gram del LCR se puede hacer el diagnóstico de forma precoz, como sucedió en este caso, en el que se encontró un bacilo gram positivo intracelular que fue identificado como L. monocytogenes. La ampicilina con o sin un aminoglucósido es el tratamiento de elección para la L. monocytoge nes y en el caso de los pacientes inmunodeprimidos la duración del mismo no debe ser inferior a 3-6 semanas $(2,3,8,13)$.

En el segundo paciente la evolución fue mortal en un corto periodo de tiempo y la presencia de la listeria no se sospechó antes de su fallecimiento. La presentación como bacteriemia $(2,3,9)$ es la más frecuente en los enfermos con infección por VIH y, aunque pueden existir localizaciones atípicas $(11,14,15)$, la afectación peritoneal es excepcional.

Aunque algunos autores han encontrado un mayor riesgo de listeriosis en los enfermos con infección por VIH $(6,16)$,

TABLA I

EVOLUCIÓN DE LOS PARÁM ETROS DEL LÍQ UIDO CEFALORRAQUÍDEO EN EL TRANSCURSO DE LA ENFERM EDAD DEL PACIENTE DEL CASO N요

\begin{tabular}{|c|c|c|c|c|c|}
\hline & $\begin{array}{c}\text { Recuento } \\
\text { celular } \\
\text { (cels/ } \mathrm{mm}^{3} \text { ) }\end{array}$ & $\begin{array}{l}\text { Tipo } \\
\text { celular }\end{array}$ & $\begin{array}{c}\text { Glucosa } \\
\text { LCR/Sangre }\end{array}$ & $\begin{array}{c}\text { Proteínas } \\
\text { en LCR } \\
\end{array}$ & $\begin{array}{c}\text { Tratamiento } \\
\text { utilizado }\end{array}$ \\
\hline & & & & & Ampicilina \\
\hline Pre-tto & 2000 & $80 \%$ PM N & 38 mg\% (99) & $1,40 \mathrm{~g} / \mathrm{L}$ & $\begin{array}{c}+ \\
\text { Cefotaxima }\end{array}$ \\
\hline Semana 1 & 450 & $95 \%$ LF & 28 mg\% (81) & $1,86 \mathrm{~g} / \mathrm{L}$ & $\begin{array}{l}\text { Toxicodermia* } \\
\text { Cotrimoxazol }\end{array}$ \\
\hline Semana 2 & 100 & $90 \%$ LF & $39 \mathrm{mg} \%(90)$ & $1,09 \mathrm{~g} / \mathrm{l}$ & Cotrimoxazol \\
\hline Semana 4 & 80 & $90 \%$ LF & $43 \mathrm{mg} \%(84)$ & 0,63 & Cotrimoxazol \\
\hline
\end{tabular}

Pre-tto: pre-tratamiento. PM N: polimorfonucleares. LF: linfocitos. *Por la aparición de toxicodermia se suspendieron la ampicilina y la tobramicina. 
por razones desconocidas, los pacientes con sida son menos susceptibles a la infección por Listeria monocytogenes que otros sujetos inmunodeprimidos. Sin embargo, no hay que olvidar que puede ser responsable de cuadros de meningitis y/o sepsis de evolución potencialmente fatal.

\section{Bibliografía}

1. Southwick FS, Purich DL. Mechanisms of disease: intracelular pathogenesis of listeriosis. N Engl J Med 1996; 334: 770-776.

2. Decker CF, Simon GL, DiGiogia RA, Tuazon CU. Listeria monocytogenes infections in patients with AIDS: report of five cases and review. Rev Infect Dis 1991; 13: 413-417.

3. Berenguer J, Solera J, Díaz MD et al. Listeriosis in patients with human inmunodeficiency virus. Rev Infect Dis 1991; 13: 115-119.

4. Bouvet E, Suter F, Gibert C, Witchitz JL, Bazin C, Vachon F. Severe meningitis due to Lysteria monocytogenes: a review of 40 cases in adults. Scand J Infect Dis 1982; 14: 267-270.

5. Aladro Y, Pérez SE, Elcuaz R, Moreno A, Granados R. Listeriosis en adultos no gestantes durante un brote epidémico en Gran Canaria. Rev Clin Esp 1995; 195: 154-9.

6. Ewert DP, Lieb L, Hayes PS, Reeves MW, Mascola L. Listeria monocytogenes infection and serotype distribution among HIV-infected persons in Los Angeles Country, 1985-1992. J Acquir Immune Defic Syndr Hum Retrovirol 1995; 8: 461-465.

7. Durand ML, Calderwood SB, Weber DJ et al. Acute bacterial meningitis in adults: a review of 493 episodes. N Engl J Med 1993; 328: 21-28.

8. Kales CP, Holzman RS. Listeriosis in patients with HIV infection: clinical manifestations and responses to therapy. J Aquir Immune Defic Syndr 1990; 3: 139-143.

9. Mascola L, Lieb L, Chiu J, Fanin SL, Linnan MJ. Listeriosis: an uncommon opportunistic infectionin patients with acquired immunodeficiency syndrome. A report of five cases and a review of the literature. Am J Med 1988; 84: 162-164.

10. Harvey RL, Chandrasekar PH. Chronic meningitis caused by Listeria in a patient infected with human immunodeficiency virus. J Infect Dis 1988; 157: 1091-1092.

11. Riancho JA, Echevarría S, Napal J, Martín Duran R, González Macía J. Endocarditis due to Listeria monocytogenes and human immunodeficiency virus infection. Am J Med 1988; 85: 737.

12. Angulo FJ, Swerdlow DL. Bacterial enteric infections in persons infected with human immunofdeficiency virus. Clin Infect Dis 1995; 21: S84-93.

13. Roldán A, Gutiérrez A, Jimenez J, Agulla A, Zancada F, Fernández A. Meningitis por Listeria e infección por VIH. Rev Clin Esp 1991; 188: 197-198.

14. Vázquez A, Ramos JM, Pacho E, Rodriguez Pérez A, Cuenca Estrella M, Esteban J. Cutaneous listeriosis in a patient infected with the human immunodeficiency virus. Clin Infect Dis 1994;19: 988-989.

15. Milgrim LM, Rubin JS, Rosenstreinch DL, Small CB. J Otolaryngol 1994; 234: 450-453.

16. Jurado RL, Farley MM, Pereira E et al. Increrased risk of meningitis and bacteriemia due to Listeria monocytogenes in patients with human immunodeficiency virus infection. Clin Infect Dis 1993; 17: 224-227. 